\title{
COMPORTAMENTO HIDROLÓGICO EM UMA ÁREA DE MOVIMENTO DE MASSA NA BACIA DO RIO SÃO PEDRO- NOVA FRIBURGO/RJ.
}

\author{
Mariana Gomes Ferreira $^{(1)}$, Juliana Martins de Souza ${ }^{(2)}$, Ana Valéria Freire Allemão Bertolino ${ }^{(3)}$ \\ (1) Departamento de Geografia/Faculdade, Universidade do Estado do Rio de Janeiro/FFP, E-mail: \\ marianag.ferreira@hotmail.com \\ (2) Departamento de Geografia/Faculdade, Universidade Federal do Rio de Janeiro. E-mail: \\ souza.julianamartins@gmail.com \\ (3) Departamento de Geografia/Faculdade, Universidade do Estado do Rio de Janeiro/FFP, E-mail: \\ anabertolino@uol.com.br
}

\section{Eixo: SISTEMA GEOMORFOLÓGICOS: ESTRUTURA, DINÂMICAS E PROCESSOS}

\begin{abstract}
Resumo
Uma gama de eventos pode estar relacionada a deflagração de um movimento de massa, mas dentre os diversos fatores ligados a este processo, destaca-se o papel hidrológico. Neste sentido, esta pesquisa busca analisar a dinâmica hídrica de uma cicatriz localizada na bacia do rio São Pedro; no distrito de São Pedro da Serra, Nova Friburgo para diagnosticar o movimento da água no solo através do monitoramento de campo. Foram utilizados blocos de matriz granular (GMS) em profundidades de 15,45 e $60 \mathrm{~cm}$, em diferentes posições topográficas. Os resultados demonstram comportamentos hidrológicos distintos posições e profundidades. Analisando os dados pode-se perceber que o período apresentou médias de $133.84 \mathrm{~mm}$ de precipitação, e o mês de dezembro apresentou o maior índice de precipitação; $480.0 \mathrm{~mm}$, a medida que agosto obteve apenas $29.91 \mathrm{~mm}$, período mais seco, mas os valores de $\mathrm{kPa}$ em profundidade $(45 \mathrm{~cm} / 60 \mathrm{~cm})$ permaneceram próximos a saturação com $-31 \mathrm{kPa}$.
\end{abstract}

Palavras chave: propriedades hídricas, movimento de massa.

\section{Introdução.}

Não é de hoje que existem estudos evidenciando os eventos de chuvas como agente detonador do processo de movimento de massa, entretanto este é apenas um no conjunto de diversos fatores que podem acelerar ou desencadear tal processo. Causando até mesmo complexidade na classificação dos tipos de movimento de massa de acordo com Fernandes \& Amaral (2001), visto as rápidas transformações que ocorrem nas cicatrizes.

Movimentos de massa é um termo utilizado para definir de forma geral todos os movimentos de massa ao longo de uma encosta de uma massa de rocha, solo ou detritos por efeito da força gravitacional (CRUDEN, 1991). E constituem um importante processo natural que atua na dinâmica de vertente e é definido como movimento de solo, rocha e/ou vegetação ao longo da 
vertente sob ação direta da gravidade (TOMINAGA, 2011). Onde a contribuição de outro meio, de água ou gelo se dá pela redução da resistência dos materiais de vertente e/ ou pela indução do comportamento plástico e fluido dos solos.

Para Bloom (1988) a água em movimento não está excluída desse processo, sua presença tem função. É necessário ter o conhecimento das rotas preferenciais dos fluxos tanto superficiais quanto subsuperficiais para obter maior compreensão da dinâmica exercida, uma vez que formas do relevo irão definir os níveis de convergência hidrológica de uma determinada topografia, bem como os níveis de poro-pressão (FERNANDES E AMARAL, 2001).

Por isso, vê-se a importância da mensuração da umidade do solo, já que a dinâmica da água tem um papel importante nos deslizamentos. E neste sentido, os blocos de matriz granular (GMS) são apresentados como equipamentos apropriados à este tipo de monitoramento (SHOCK,1988). Os potenciais matriciais consistem em pequenos blocos que permitem a medição da condutividade elétrica do solo, realizado a partir de dois eletrodos envolvidos por uma matriz granular.

Tendo esta base, o trabalho consiste na descrição e compreensão dos valores de potenciais matriciais e seu comportamento no período amostral de um ano, em uma cicatriz localizada na bacia do Rio São Pedro, em São Pedro da Serra, distrito do município de Nova Friburgo a partir do monitoramento de campo, com a instalação de sensores de matriz granular em distintas profundidades da topografia.

\section{2. Área de Estudo.}

A pesquisa foi realizada na Bacia do Rio São Pedro em São Pedro da Serra, distrito do município de Nova Friburgo no Rio de Janeiro na região serrana do estado. A bacia apresenta $23 \mathrm{~km}^{2}$ e se encontra em um bioma de Mata Atlântica, numa área de Proteção Ambiental chamada Macaé de Cima conforme o mapa (Figura 1): 


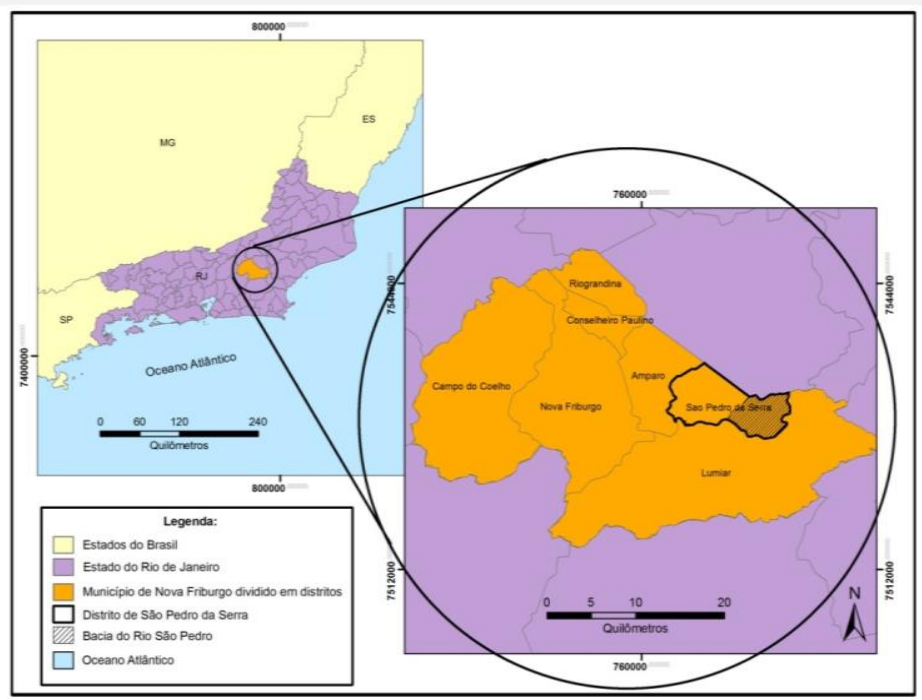

Figura 1: Localização do município de Nova Friburgo, destacando a bacia do rio São Pedro. (Fonte: Souza 2014)

\section{Metodologia do Trabalho.}

\subsection{Monitoramento da Pluviosidade.}

O monitoramento pluviométrico foi possível a partir da estação Meteorológica Automatizada, um modelo THIES TLX-MET (Figura 2), responsável pela coleta dos dados de precipitação a cada 10 minutos. A estação está instalada dentro de uma propriedade de agricultura familiar (SOUZA, 2014), que possui um registro diário, cujos os dados são organizados pela técnica responsável.

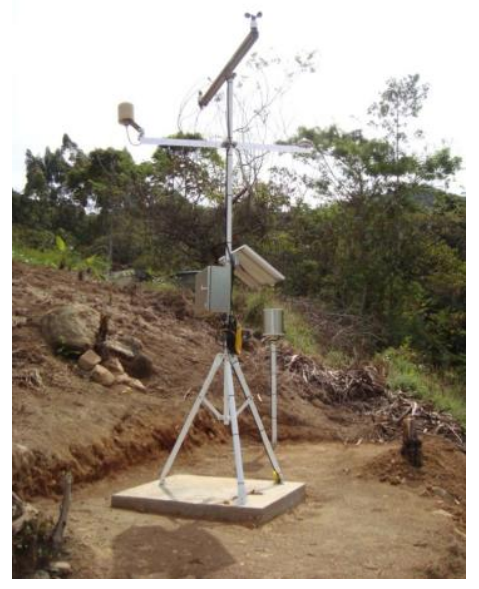

Figura 2: Estação Automatizada THIES TLX-MET utilizada na obtenção de dados climatológicos próximos a área de estudo. Fonte Souza, 2014. 


\subsection{Potencias Matriciais}

A análise das cicatrizes foi possível através do monitoramento de baterias de sensores de matriz granular, visto que de acordo com Shock (1998), como citado por Souza (2014) estes instrumentos não necessitam de fluxagem, diferente de outros métodos comumente utilizados como os tensiômetros, mas é necessário a mensuração diária através do aparelho Watermak ${ }^{1}$. Após a identificação, foram instalados 9 baterias nas profundidades de $15 \mathrm{~cm}, 45 \mathrm{~cm}$ e $60 \mathrm{~cm}$, em topografias distintas, ou seja no topo da cicatriz e nas laterais, ao lado esquerdo e ao lado direito nas respectivas profundidades como pode ser analisado no esquema (Figura 3):

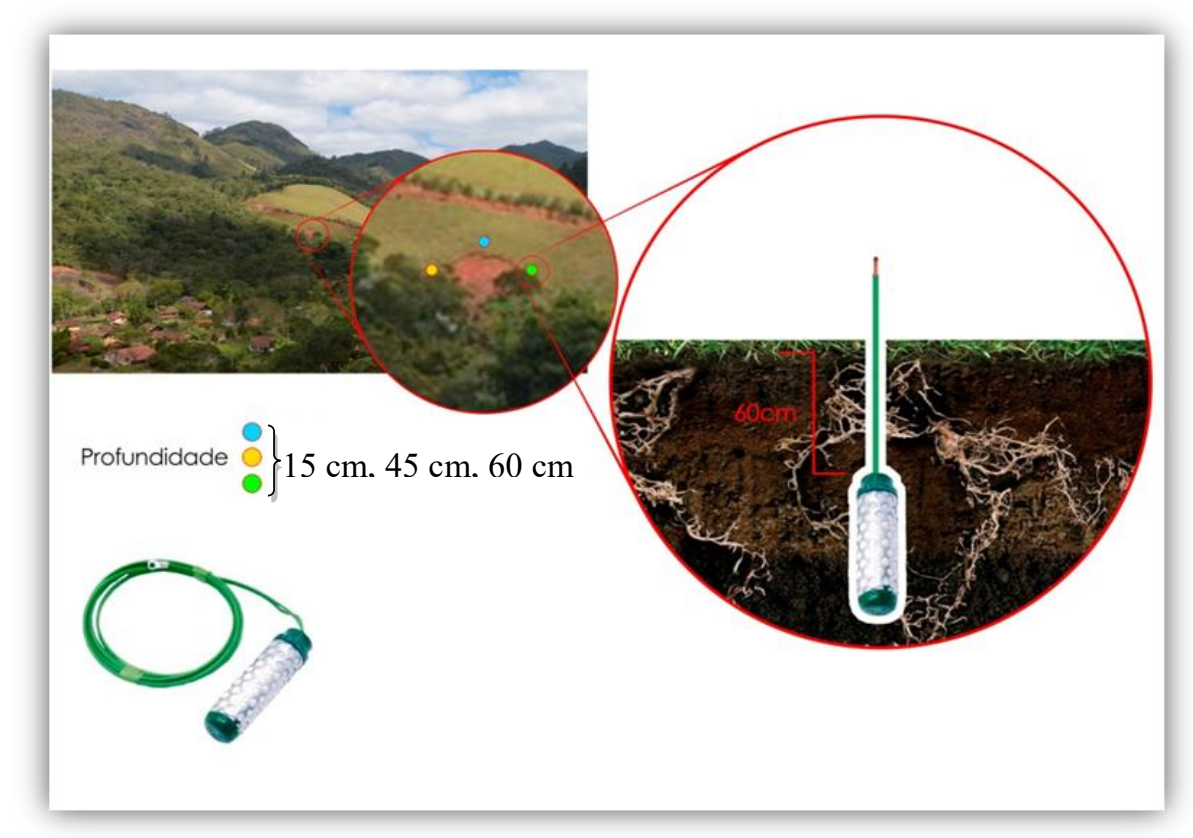

Figura 3 - Localização dos sensores de matriz granular (GMS) no topo e nas laterais da cicatriz nas distintas profundidades.

\section{Resultados e discussões}

Para melhor compreensão dos valores de potenciais matriciais, foi realizado um levantamento das precipitações mensais (tabela I). O mês de dezembro apresenta-se como o mês mais úmido, com o maior índice $(480 \mathrm{~mm})$, em contrapartida o mês mais seco é agosto, com precipitação total de

\footnotetext{
${ }^{1}$ The Watermark (Irrometer Company, Riverside, CA) is a granular matrix sensor, similar to a gypsum block. It is consist of two concentric electrodes embedded in a reference matrix material, which is surrounded by a synthetic membrane for protection against deterioration.
} 


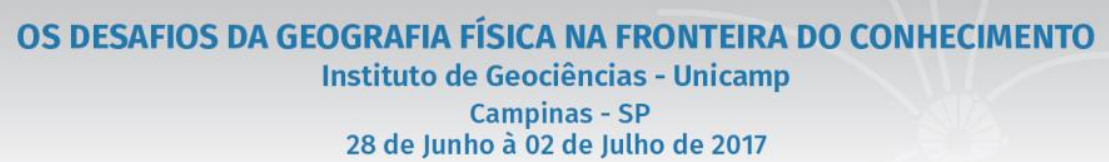

$29.91 \mathrm{~mm}$. Este comportamento é típico da área de estudo e já foi relatado por Souza 2014 (Figura 4).

Tabela I - Valores de precipitação (mm) do período de novembro de 2013 a novembro de 2014.

\begin{tabular}{|c|c|}
\hline Meses 2013/2014 & Soma de Chuva(mm) \\
\hline Novembro & 237.5 \\
\hline Dezembro & 480.0 \\
\hline Janeiro & 46.1 \\
\hline Fevereiro & $\mathbf{6 4 . 7}$ \\
\hline Março & $\mathbf{1 2 3 . 9}$ \\
\hline Abril & $\mathbf{2 1 7 . 1 9}$ \\
\hline Maio & 37.83 \\
\hline Junho & 43.31 \\
\hline Julho & 43.69 \\
\hline Agosto & 29.91 \\
\hline Setembro & 33.24 \\
\hline Outubro & $\mathbf{7 2 . 0 5}$ \\
\hline Novembro & $\mathbf{2 2 0}$ \\
\hline
\end{tabular}

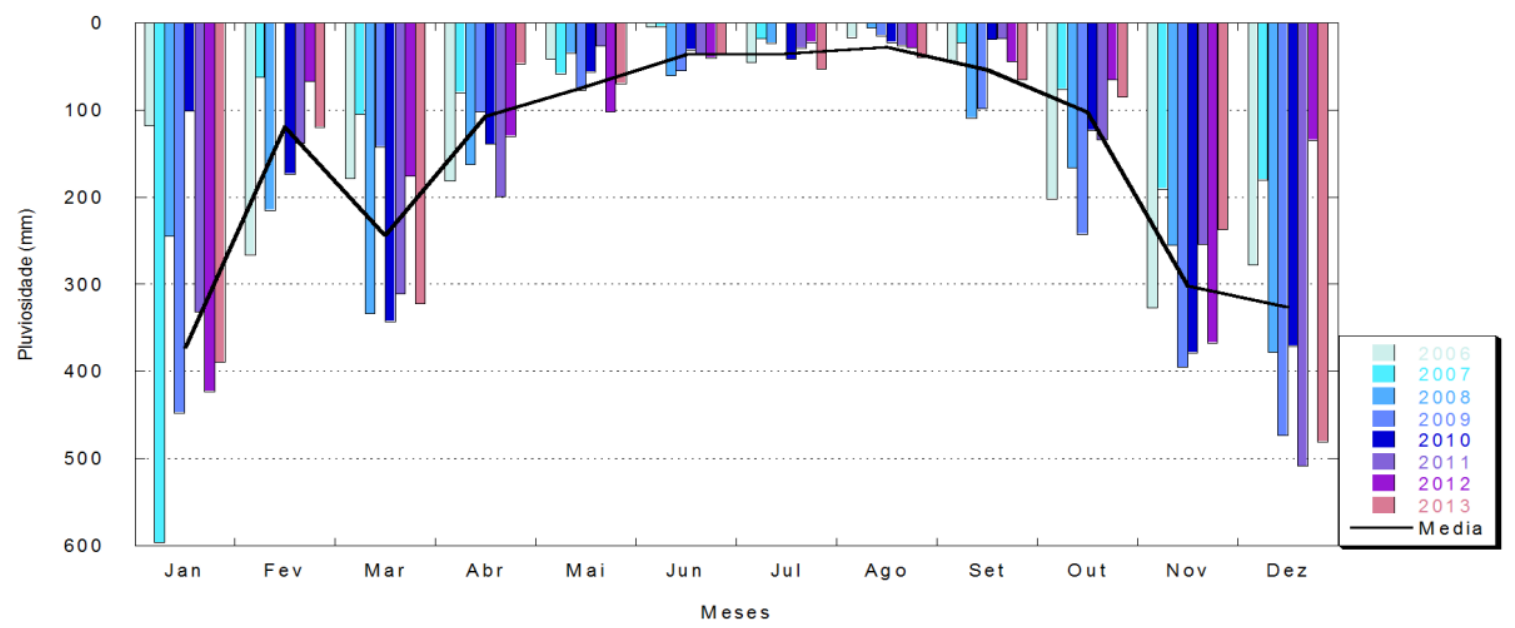

Figura 4 - Total da precipitação mensal de 2006 a 2013, com a média de 2006 a 2013. (Fonte: Souza 2014)

A partir da análise das precipitações (Tabela I), relacionada as médias de potenciais matriciais (Tabela II), percebe-se que o mês de novembro de 2013 apresentou o maior índice de pluviosidade $237,5 \mathrm{~mm}$, logo as profundidades de $15 \mathrm{~cm}$ e $45 \mathrm{~cm}$ ficaram próximas a saturação, com médias de $-18 \mathrm{kPa}$ e $-10 \mathrm{kPa}$ (tabela II). O mês seguinte obteve o maior índice de 
XVII Simpósio Brasileiro de Geografia Fisica Aplicada

I Congresso Nacional de Geografia Física
OS DESAFIOS DA GEOGRAFIA FÍSICA NA FRONTEIRA DO CONHECIMENTO

Instituto de Geociências - Unicamp

Campinas - SP

28 de Junho à 02 de Julho de 2017

precipitação, no entanto perdeu umidade em superfície, enquato as demaisprofundidades permaneceram próximas à saturação. Como também pode ser visualizado no gráfico (Figura 5).

Tabela II: Tabela com as médias de potencial matricial por profundidade do mês de novembro de 2013 até novembro de 2014.

\begin{tabular}{|c|c|c|c|c|c|c|c|c|c|c|c|c|c|}
\hline Prof. & Nov & Dez & Jan & Fev & Mar & Abr & Mai & Jun & Jul & Ago & Set & Out & Nov \\
\hline $\begin{array}{c}\mathbf{1 5 c m}( \\
\mathbf{k P a})\end{array}$ & -18.0 & -51.0 & -46.0 & -108.0 & -32.0 & -17.0 & -25.0 & -25.0 & -33.0 & -30.0 & -59.0 & -53.0 & -31.0 \\
\hline $\begin{array}{c}\mathbf{4 5 c m}( \\
\mathbf{k P a})\end{array}$ & -10.0 & -16.0 & -13.0 & -50.0 & -50.0 & -22.0 & -23.0 & -23.0 & -26.0 & -31.0 & -32.0 & -49.0 & -37.0 \\
\hline $\begin{array}{c}\mathbf{6 0} \mathbf{c m} \\
\mathbf{( k P a})\end{array}$ & -39.0 & -27.0 & -20.0 & -42.0 & -37.0 & -20.0 & -18.0 & -21.0 & -32.0 & -31.0 & -24.0 & -51.0 & -40.0 \\
\hline
\end{tabular}

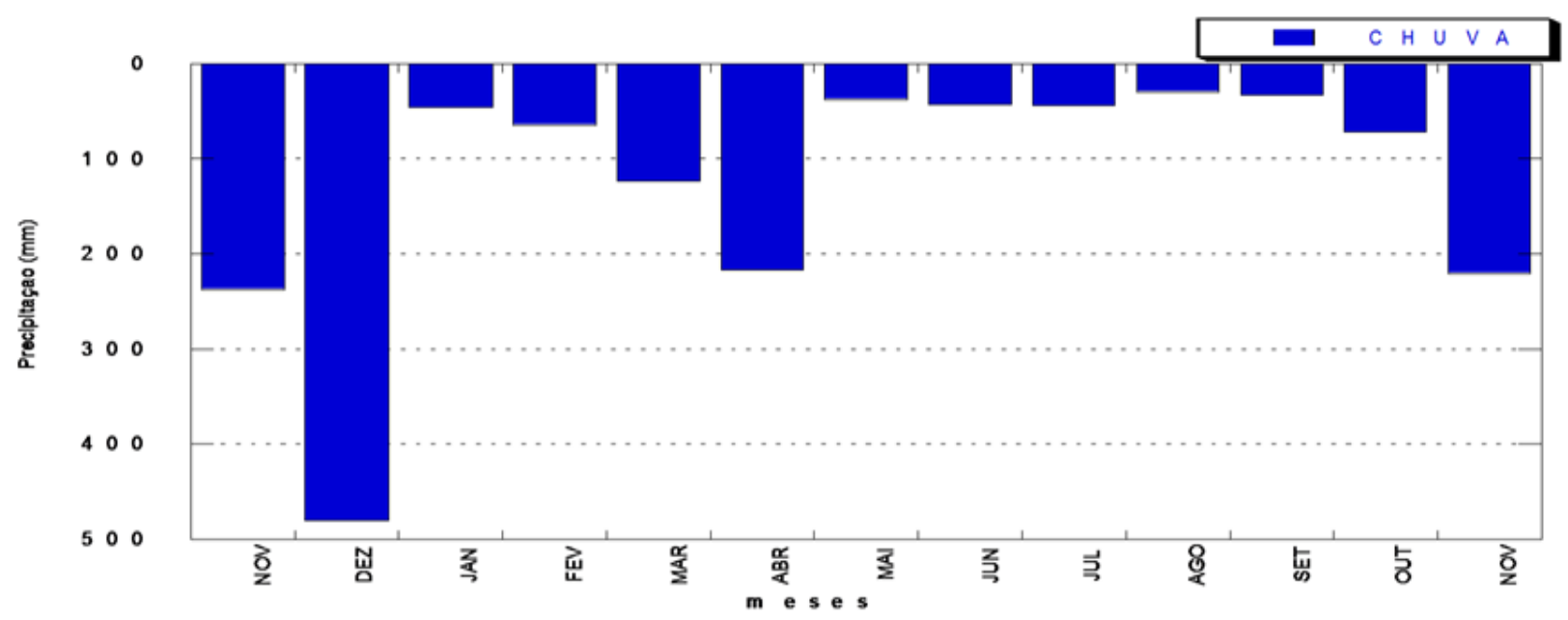

Figura 5 - Variação de precipitação de novembro de 2013 a novembro de 2014.

Para melhor compreensão também foi elaborado gráficos com as médias dos potenciais matriciais por profundidade e comparados com as precipitações, com o intuito de obter um parâmetro geral destes valores e observar de forma abrangente os distintos comportamentos da água no solo. 


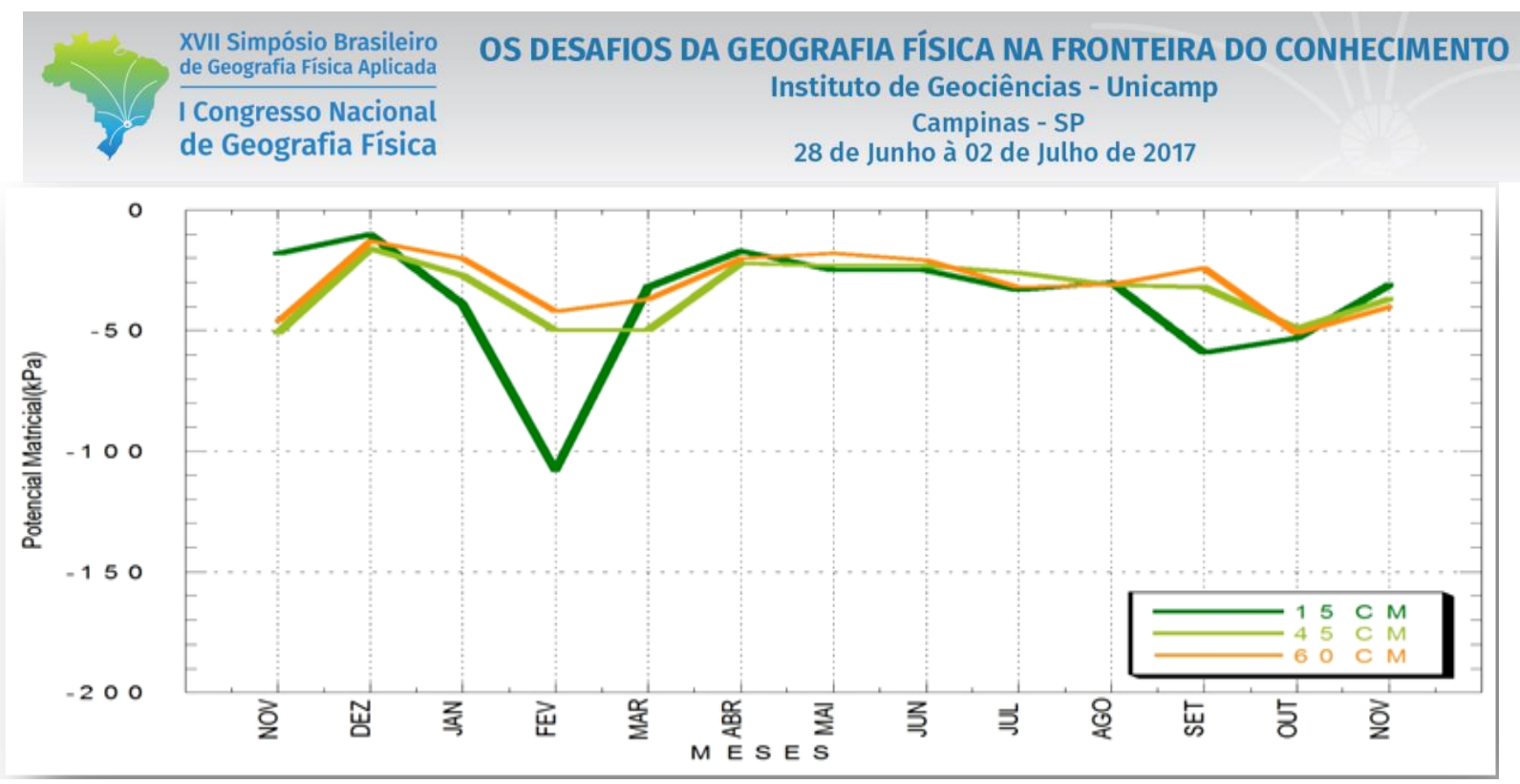

Figura 6 - Média dos potenciais matriciais dos GMS's até -200 kPa nas diferentes profundidades de novembro de 2013 até novembro de 2014

De acordo com o gráfico de precipitação (Figura 5), observa-se um alto índice de precipitação nos meses de novembro e dezembro de 2013, onde este último ultrapassou os $400 \mathrm{~mm}$, à medida que os meses de janeiro à março apresentaram somas inferiores a $100 \mathrm{~mm}$, já o mês de abril obteve pluviosidade de $220 \mathrm{~mm}$, em seguida perceba o período de maio à outubro, os valores permaneceram abaixo de $50 \mathrm{~mm}$.

Ao realizar a análise do gráfico de potencial matricial (Figura 6), tem-se que no mês de novembro a superfície em $15 \mathrm{~cm}$ apresentou média de $-20 \mathrm{kPa}$, a medida que as profundidades de $45 \mathrm{~cm}$ e $60 \mathrm{~cm}$ apresentaram médias entre $-40 \mathrm{kPa}$ e $-50 \mathrm{kPa}$. No mês seguinte, os potenciais matriciais sofrem aumento, mostrando maior retenção de umidade. E a superfície permanece saturada, com valor de média de kPa maiores que em subsuperfície. Em seguida percebe-se um período de mais seco, como pode ser visto no gráfico (Figura 5). Onde no mês de janeiro, como pode ser observado gráfico (Figura 6), apresentando há uma retenção de umidade a medida que superfície $(15 \mathrm{~cm})$ apresenta perdas maiores de umidade.

O mês de fevereiro também apresenta pouca pluviosidade, e ao visualizar o gráfico (Figura 5) mais uma vez, tem-se uma superfície com perda de água, com média de -100 kPa, mas observa-se o resultado nas outras profundidades no mesmo gráfico: também existe uma perda,no entanto, muito menor quando comparada a superfície, pois estão entre $-40 \mathrm{kPa}$ e $-50 \mathrm{kPa}$. Prosseguindo com a análise, tem-se o mês de março, onde este obteve soma de precipitação superior à $100 \mathrm{~mm}$, logo refletidos nos dados de potenciais matriciais, quando observada a superfície $(15 \mathrm{~cm})$, um 
crescimento brusco deste valor superando as demais profundidades quanto a presença de umidade. Entretanto estas últimas permanecem com os valores de médias de $\mathrm{kPa}$ bem próximos do mês anterior (fevereiro), demonstrando a partir desse momento uma retenção de água, principalmente em $60 \mathrm{~cm}$. Logo no mês seguinte (abril), há um aumento dos índices de precipitação, com soma maior que $200 \mathrm{~mm}$, com isso os dados de potenciais matriciais demonstraram valores entre $-20 \mathrm{kPa}$ e $-30 \mathrm{kPa}$, e a profundidade de $15 \mathrm{~cm}$ se sobrepõe. Além de observar um aumento significativo em $45 \mathrm{~cm}$ e $60 \mathrm{~cm}$, haja vista o diagnóstico de retenção de água em profundidade.

Já no mês de maio observa-se uma diminuição de kPa para a superfície e sub superfície, todavia a profundidade de $60 \mathrm{~cm}$ permanece acima de $-20 \mathrm{kPa}$, um período de pouca precipitação. Os meses de junho e julho apresentaram pouca chuva, mas no último vê-se a profundidade de $45 \mathrm{~cm}$ apresentando maior saturação à medida que a profundidade de $60 \mathrm{~cm}$ sofre uma pequena diminuição, possuindo valores de potencial matricial similar que a superfície. Em agosto as mesmas apresentaram valores na faixa de -20 á $-40 \mathrm{kPa}$, sem mudanças de relevância. Entretanto, o mês de setembro se destaca, pois demonstra mais uma vez a retenção de umidade na profundidade de $60 \mathrm{~cm}$, mesmo com pouca precipitação e um decréscimo da média de $\mathrm{kPa} e m$ $15 \mathrm{~cm}$. Nos meses finais do monitoramento percebe-se um maior índice de precipitação em que os potenciais matriciais tendem à saturação.

Com isso, compreende-se as médias dos valores de $\mathrm{kPa}$ por profundidade, mas à frente será descrito as médias por profundidade e topografia, pois existe distintos comportamentos quanto à topografia. Como poderá ser apreciado nos gráficos seguintes (Figuras 7, 8 e 9). 

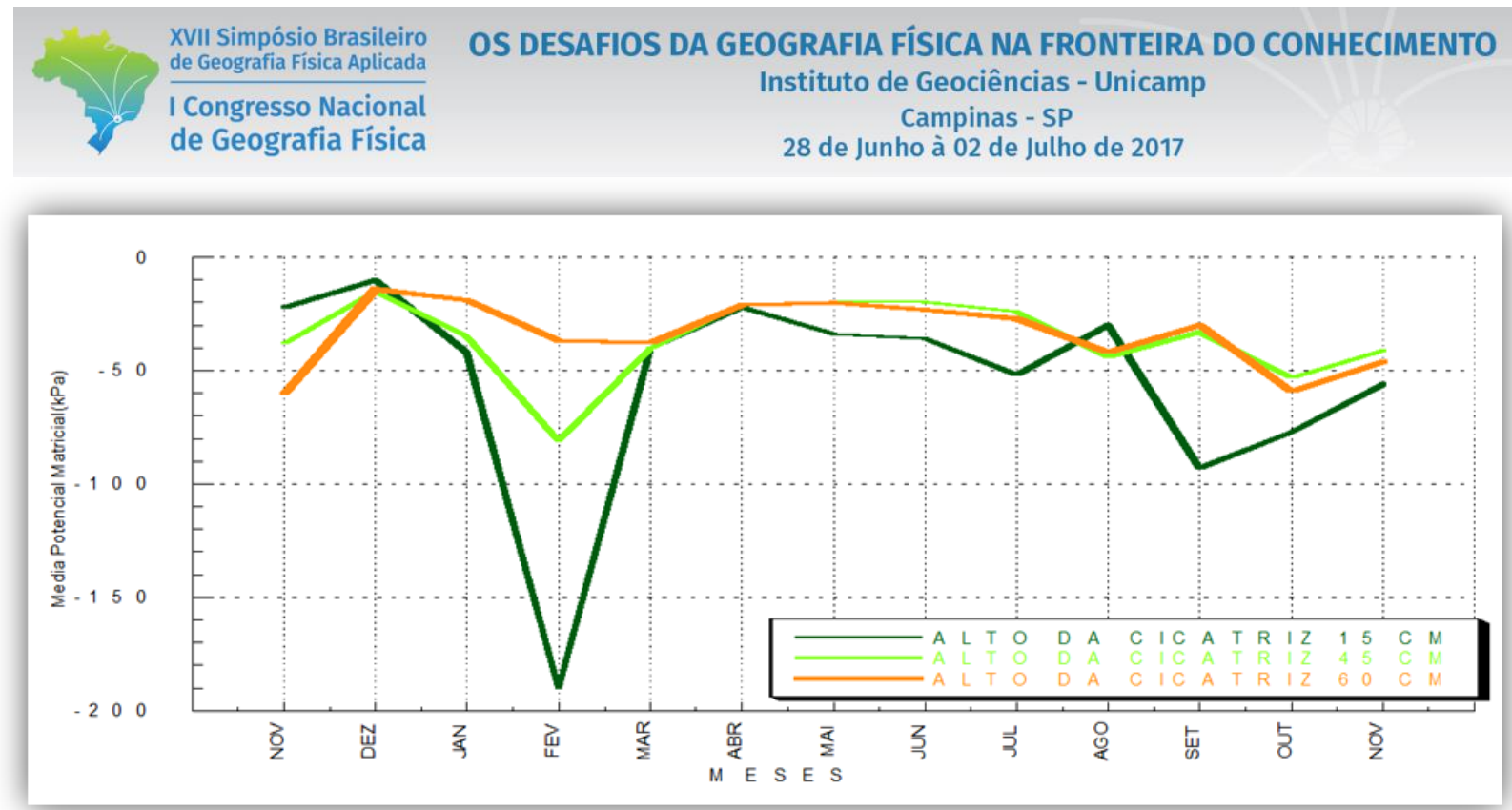

Figura 7 - Médias dos potenciais matriciais no alto da cicatriz no período de novembro de 2013 a novembro de 2014.

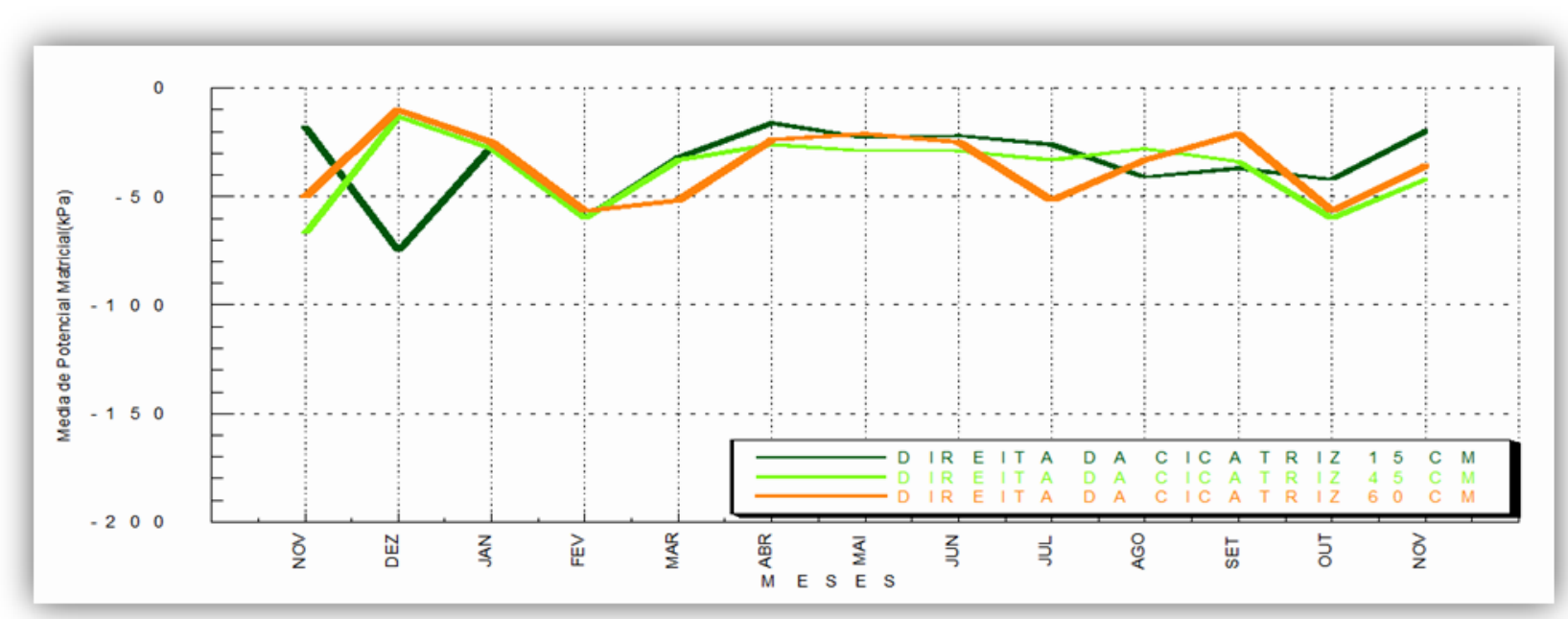

Figura 8 - Médias dos potenciais matriciais à direita da cicatriz no período de novembro de 2013 a novembro de 2014. 


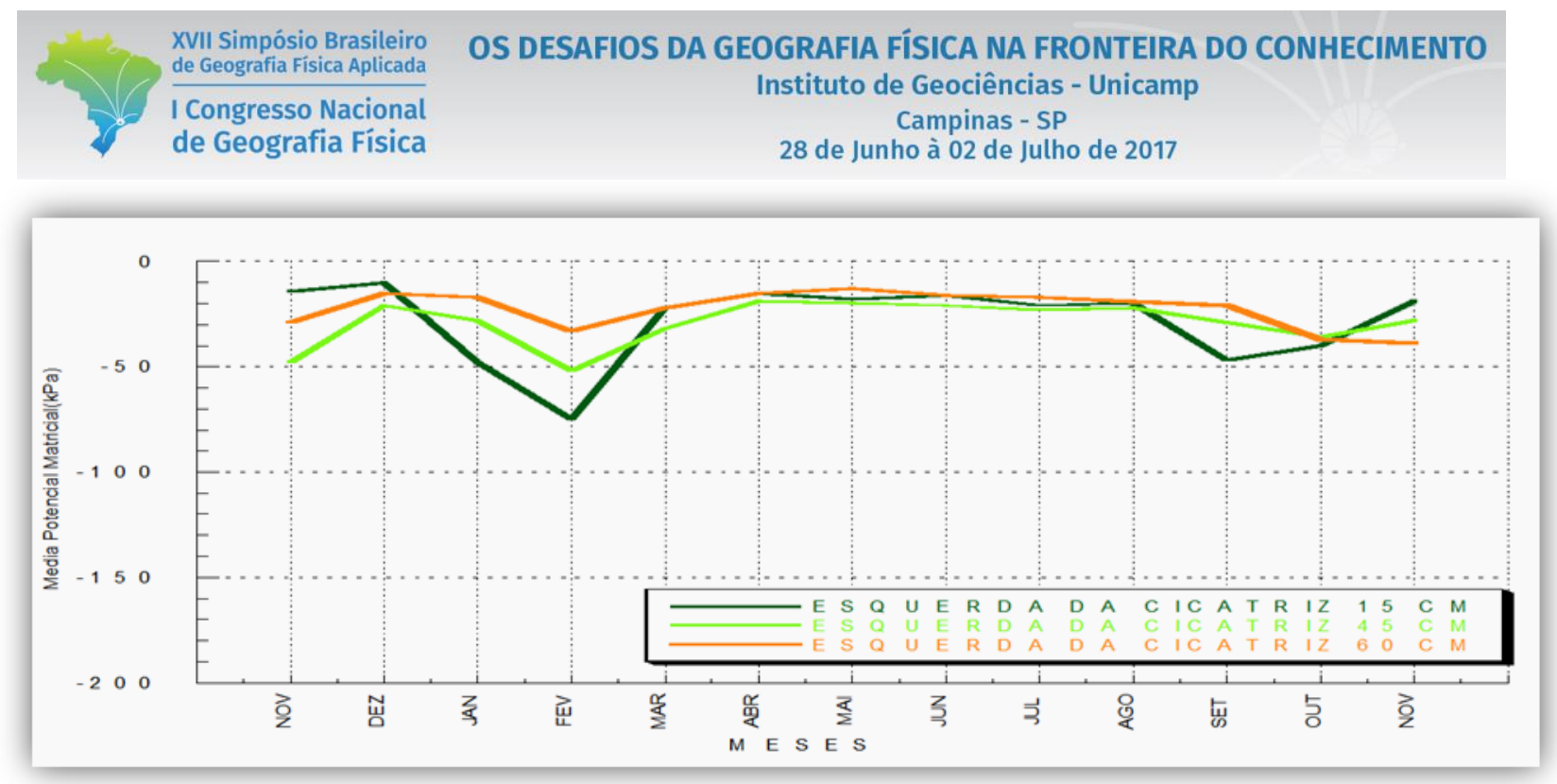

Figura 9 - Médias dos potenciais matriciais àesquerda da cicatriz no período de novembro de 2013 a novembro de 2014.

Ao comparar o potencial matricial dos gráficos referentes às distintas topografias no mês de novembro, percebe-se que as distintas profundidades demonstram comportamentos similares, entretanto na direita e na esquerda da cicatriz a profundidade de $60 \mathrm{~cm}$ obteve maior saturação. No mesmo intervalo no alto da cicatriz a profundidade de $45 \mathrm{~cm}$ chega a $-50 \mathrm{kPa}$ e a profundidade de $60 \mathrm{~cm}$ à $-30 \mathrm{kPa}$ na esquerda desta.

Já no mês de dezembro, a diferença é verificada no gráfico de potencial matricial da direita (Figura 8), a superfície $(15 \mathrm{~cm})$ apresenta uma perda de umidade, diferentemente do alto e da esquerda da encosta monitorada. Enquanto a profundidade $(60 \mathrm{~cm} / 45 \mathrm{~cm})$ tem valores acima de $50 \mathrm{kPa}$ no mesmo intervalo de tempo. Seguindo a análise dos resultados de $\mathrm{kPa}$, tem-se o mês de janeiro, onde o alto da cicatriz demonstra maior perda de umidade, mas as profundidades de $45 \mathrm{~cm}$ e $60 \mathrm{~cm}$ têm perdas, acima de $-80 \mathrm{kPa}$ ou seja menor. Mas, nas posições laterais também tem perdas, e na posição esquerda os valores de GMS's foram respectivamente $-48 \mathrm{kPa}$ para superfície, $-28 \mathrm{kPa}$ em $45 \mathrm{~cm}$ e $-17 \mathrm{kPa}$ em $60 \mathrm{~cm}$ de profundidade. Concomitante a direita da cicatriz tem valores bem próximos: $-27 \mathrm{kPa},-28 \mathrm{kPa}$ e $-25 \mathrm{kPa}$, mas que difere, pois apresenta maior saturação.

O destaque quanto à perda de água está no topo da cicatriz no mês de fevereiro. Visto o gráfico de GMS (Figura 7), onde a superfície está próxima a não saturação, no entanto em 60 cm,recarga mais lenta acima, permanecendo na próximo a saturação. Nas demais posições, apenas a 
profundidade de $15 \mathrm{~cm}$ na posição esquerda permaneceu abaixo de $-50 \mathrm{kPa}$. Quando observado o mês posterior, a posição direita se configura de forma distinta das demais, pois nesta o resultado do valor de potencial matricial para a profundidade de $60 \mathrm{~cm}$ é de $-50 \mathrm{kPa}$, no entanto nas outras topografias apresenta valores entre 0 à $-30 \mathrm{kPa}$.

O mês de abril demonstra resultados pouco distinto entre as topografia e profundidades, e também não é percebido diferenças quanto à esquerda da cicatriz nos períodos de abril à agosto, onde todas as profundidades estão próximas à saturação. Mas no alto da cicatriz nota-se no mesmo período que a profundidade de $15 \mathrm{~cm}$ tem perda de água, enquanto que tal situação é identificada no mês de julho para a profundidade de $60 \mathrm{~cm}$ (Figura 8), obtendo um aumento nos meses seguintes, agosto e setembro, ao passo que em superfície os valores de $\mathrm{kPa}$ decaem em setembro. Porém, na posição esquerda o valor da média de potencial matricial para superfície permanece quase o mesmo do mês anterior, ou seja, não apresentando perdas.

Nos meses consecutivos, verifica-se que os dados de potenciais matriciais nos gráficos (Figuras 7,8 e 9) aponta um pequeno aumento, contudo o comportamento hídrico difere nas profundidades e topografias. No alto da cicatriz a profundidade de $45 \mathrm{~cm}$ aparece com maior saturação, subsequente as profundidades de $60 \mathrm{~cm}$ e $15 \mathrm{~cm}$ respectivamente. Mas no gráfico (Figura 8), a superfície que exibe aproximação à $0 \mathrm{kPa}, \operatorname{logo}$ depois $60 \mathrm{~cm}$ e $45 \mathrm{~cm}$, já no terceiro gráfico a superfície permanece com maior potencial matricial, mas em seguida nota-se a profundidade $45 \mathrm{~cm}$ e não $60 \mathrm{~cm}$, como ocorrido nas situações anteriores.

\section{4- Conclusão}

Após a análise dos dados obtidos pelos blocos de matriz granular (GMS's) nas distintas topografias e assim relacionados aos valores de precipitação, verificou-se que há uma diferença na frente de umedecimento entre as distintas profundidades $(15 \mathrm{~cm}, 45 \mathrm{~cm}$ e $60 \mathrm{~cm})$. Já a profundidade de $15 \mathrm{~cm}$ comporta-se de forma coerente, mas apresenta uma significativa perda de umidade, quando não recebe recarga hídrica, entretanto nas profundidades de $45 \mathrm{~cm}$ e $60 \mathrm{~cm}$ a mesma fica mais tempo retida. Esta característica também é explicada por Toll (2001), visto que a chuva antecedente tem uma função importante quando relacionados às respostas das medidas de poropressão durante um evento chuvoso. Dado que quando seus valores iniciais se encontram elevados, uma forte precipitação tem a capacidade de imprimir apenas uma pequena mudança na 
medida da poropressão. Por outro lado, quando as medidas iniciais de poropressão encontram-se baixas, um pequeno evento chuvoso é o bastante para proporcionar uma grande mudança em seus valores.

Outra questão que vale ressaltar é a recarga de água que pode justificar essa umidade mais tempo retida principalmente na profundidade de $60 \mathrm{~cm}$. A água drenada ao atingir tal profundidade fica mais tempo estocada que nas demais profundidades estudadas. Como também perceber-se que o comportamento hídrico nas profundidades da encosta em análise correlaciona-se diretamente com os eventos pluviométricos na região.

\section{Bibliografia}

AMARAL, R. Desastres naturais: Conhecer para prevenir. Instituto Geológico: São Paulo, 2011.196 p.

BLOOM, A. L. Superfície da Terra. Trad. Petri, Setembrino; Ellert, Reinholt. São Paulo: Bucher;EDUSP, 1970.

CRUDEN, D.M. A Simple Definition of a Landslide. Bulletin of the International Association of Engineering Geology, 43: 27-29. 1991.

ELDREDGE, E.P., SHOCK, C.C., STIEBER, T.D., 1993. Calibration of granular matrix sensors for irrigation management. Agron. J. 85, 1228-1232.

FERNANDES, N. F., Condicionantes Geomorfológicos dos Deslizamentos nas Encostas: Avaliação de Metodologias e Aplicação de Modelo de Previsão de Áreas Susceptíveis; Revista Brasileira de Geomorfologia, volume $2, \mathrm{n}^{\mathrm{o}} 1$ (2001)51-70.

SHOCK, C.C., BRNUM, J.M., SEDDIGH, M., 1998. Calibration of watermark soil moisture sensors for irrigation management. Int. Irrig. Show, San Diego, CA, USA, pp. 139-146.

SOUZA, J. M. Características do meio físico em um escorregamento de São Pedro da Serra e suas influências nas transformações da paisagem em Nova Friburgo - RJ. 2014. Dissertação (Mestrado em Geografia) - Pontifícia Universidade Católica do Rio de Janeiro

SOUZA, J. M., ASEVEDO, L. A., SOARES,I. P., BERTOLINO, A. V. A.;Movimentos de Massa na Bacia do Rio São Pedro, município de Nova Friburgo, associado aos eventos de chuva de Janeiro de 2011 ACTA Geográfica, Boa Vista, Ed. Esp. Climatologia Geográfica, 2012. pp.149-163

TOLL, D. G., TSAPARAS, I.; RAHARDJO,H. The influence of rainfall sequences on negative pore-water pressures within slopes. Conference on Soil Mechanics and Geotechnical Engineering. ISSMGE, Istanbul, Turkey, $2,2001$. 1269-1272p.

TOMINAGA, L. K. Escorregamentos. In: TOMINAGA, L. K.; SANTORO, J.; AMARAL, R. Desastres naturais: Conhecer para prevenir. Instituto Geológico: São Paulo, 2011. 196p. 